\title{
Seitenastligatur erhöht Rezidivrate
}

Cappelli M et al. Ligation of the saphenofemoral junction tributaries as risk factor for groin recurrence. J Vasc Surg: Venous and Lym Dis 2018; 6: 224-229. Doi:10.1016/j.jvsv.2017.09.005

Trotz technischer Fortschritte sind bei der chronischen venösen Erkrankung (CVD) Rezidivcrosseninsuffizienzen häufig. Die Flush-Ligatur im Bereich der saphenofemoralen Junktion ist als postoperativer Rückfallschutz etabliert. Die Bedeutung der Seitenastligatur ist hingegen wenig untersucht und eindeutige Indikationskriterien fehlen laut Cappelli. Bei thermoablativen und Tumeszenzverfahren beeinflusste die Schonung der Seitenäste die Rezidivrate nicht. Die retrospektive Studie mit $1216 \mathrm{~Pa}$ tienten (1433 Beine) zeigte unterschiedliche Langzeitergebnisse nach einer hohen Ligatur in Abhängigkeit von einer Ligatur/ Schonung der Seitenäste.

Vollständige Daten lagen von 763 Patienten (867 Beine) mit einem Durchschnittsalter von 63 Jahren vor. Alle erhielten eine hohe Flush-Ligatur und Division der V. saphena magna. In Gruppe A (plus Seitenast- ligatur) waren 297 und in Gruppe B (Schonung der Seitenäste) 570 Beine. Postoperative Ultraschallkontrollen erfolgten nach 1 Woche, 3 Monaten, 1 Jahr und schließlich jährlich. Die Beobachtungsdauer betrug 5 Jahre (median). Die Gruppen unterschieden sich bzgl. klinischer und soziodemografischer Basisbefunde sowie der sonografischen Ausgangslage nicht wesentlich. In allen Fällen gelangen die OPs und schwere Komplikationen traten nicht ein. Ein erneuter Reflux im Bereich der saphenofemoralen Verbindung kam insgesamt bei 3,3\% vor. Die Intervalle von der OP bis zum Rückfall betrugen im Schnitt 4,5 Jahre (Gruppe A) und 3 Jahre (Gruppe B). Verglichen mit Gruppe B entwickelten Patienten mit Seitenastligatur signifikant häufiger Rezidive (7,4\% vs. 1,1\%; OR 7,52; $95 \%-\mathrm{KI}$ 2,91-22,88; $p<0,001)$. Die meisten Refluxphänomene im Bereich der Primärlokalisation beruhten auf einer Rekonnektion des
Stumpfes im Bereich der ursprünglichen Verbindung (Gruppe A: 3,7\% vs. Gruppe B: $0,2 \%$ ). Eine weitere Ursache stellten neue pelvine Shunts dar (3\% vs. 0,5\%). Neue inkompetente Perforatorvenen kamen in den Gruppen vergleichbar oft vor.

Die hämodynamischen Grundlagen der höheren Rezidivrate nach Seitenastligatur bleiben offen. Möglich sei ein „FlushingFlow“ aus den Seitenästen in den Stumpf der V. saphena magna. Dieser verhindere evtl. eine Stase, Inflammationsprozesse, eine Neoangiogenese und pathologische Scherkräfte. Prospektive Folgeuntersuchungen sollten auch die spezifische Relevanz der Einzeläste und unterschiedliche OP-Methoden berücksichtigen, so die Autoren.

Dr. med. Susanne Krome, Melle 